\title{
Sistema Multiagente Aplicado a Desafios Lógicos Abertos: Uma Possibilidade de Interação Através do Incremento Lúdico
}

\author{
Othon C. Bastos Filho1, Margarete Axt1, Sofiane Labidi2, \\ Janete S. Costa1, Paloma D. Silveira1, Cristiane T. Machado2 \\ 1 Universidade Federal do Rio Grande do Sul (UFRGS) \\ Programa de Pós-Graduação em Informática na Educação (PPGIE) \\ Laboratório de Estudos em Linguagem, Interação e Cognição (LELIC) \\ Caixa Postal: 5071 - CEP: 90041-970 Porto Alegre - RS. \\ 2 Universidade Federal do Maranhão, Laboratório de Sistemas Inteligentes, LSI/UFMA, \\ Campus do Bacanga, 65.000-000 - São Luís-MA, Brasil. \\ othonb@hotmail.com, maaxt@ufrgs.br, labidi@uol.com.br, janetesander@gmail.com, \\ palomads86@yahoo.com.br, cristianemachado@yahoo.com.br
}

\begin{abstract}
Resumo
O trabalho contextualiza uma aplicação de técnicas da IA, na abordagem de Sistemas Multiagentes, fundamentada no Método Clínico de Piaget. A pesquisa em fase de implementação, trabalha, a partir de um incremento lúdico as possibilidades de obter-se situações de aprendizagem e, conseqüentemente, de desenvolvimento cognitivo para o usuário. Isso se dará por meio de estágios e a adaptabilidade do sistema ao usuário, ou seja, uma possibilidade de interação em um sistema com proposta construtivista. O Sistema IOCS faz parte de uma plataforma-ambiente integrada entre os Laboratórios LSI/UFMA e LELIC/UFRGS e tem como objetivo geral permitir uma visualização do relacionamento entre os processos mentais cognitivos, possibilitando um suporte à docência e à formação continuada em ambientes informatizados.
\end{abstract}

Palavras-Chave: Cognição, Agentes Inteligentes, Abstração Reflexionante, Desafios Abertos.

\begin{abstract}
This work translates into words the use of AI techniques on the Multiagent Systems approach based upon the Clinical Method of Piaget. The research is in its implantation phase. It explores, using a game environment, possibilities to enter a learning situation and, consequently, a cognitive upgrading. This should happen by means of a staging and adaptable system, i.e., a possibility of interaction in a system with a constructivist proposition. System IOCS is part of an integrated environment-platform between LSI/UFMA and LELIC/UFRGS Laboratories, and generally aims at allowing a visualization of the relationship between cognitive mental processes, making possible a teachers' support and continued upgrading in IT environments.
\end{abstract}

Keywords: Cognition, Intelligent Agents, Reflective Abstraction, Open Challenges. 


\section{Introdução}

Hoje, várias instituições de ensino estão buscando a inclusão de seus alunos à educação digital. O incremento lúdico, como os jogos, tem sido uma das estratégias utilizadas para se trabalhar os problemas relacionados à aprendizagem. Os "jogos" captam atenção pelos desafios, pelas interfaces atraentes e principalmente pela característica de jogo coletivo viabilizada pela conexão em rede e as possibilidades de cooperação grupal. Com isso, tornam-se um importante recurso para atendimento a crianças com déficit de atenção entre outras dificuldades. Entretanto, os jogos de uma maneira geral não são adaptativos, ou seja, não possibilitam adaptações de ações externas e internas, já que essas ações estão previamente codificadas e compiladas.

Pensando nessa imutabilidade, observou-se a necessidade de se propor, não propriamente jogos, mas um Sistema de Desafios Abertos Inteligentes - Intelligent Open Challenges System - IOCS (Bastos Filho et al., 2004). Essa proposta está voltada para a educação, isto é, vem ao encontro de desenvolver atividades que instiguem as construções de significados estruturados por meio da interação e do componente lúdico e que tragam para os participantes possibilidades de ganho cognitivo.

O objetivo é trabalhar a aprendizagem a partir de uma concepção de construção do conhecimento, ou seja, a aprendizagem que acontece por meio de situações desequilibradoras. E, para atender a este pressuposto, utilizaremos desafios1 com regras lógicas incluídas na programação, com o aporte da tecnologia multiagentes, uma subárea da Inteligência Artificial. Espera-se desafiar os usuários à problematização, motivando-os a interagir com demais usuários de modo a construir hipóteses (pensamento dedutivo) a partir do desafio proposto pelo sistema. Como desfeche dos desafios, os usuários, além da discussão coletiva das possíveis alternativas de solução, podem socializar as soluções encontradas, deixando-as registradas, bem como interagir com vistas a propor coletivamente novas possibilidades em termos de desafios.

Os desafios estão baseados na perspectiva de estágios cognitivos, a partir das provas piagetianas. Buscam e incentivam a compreensão dos mecanismos envolvidos na construção de conhecimento. No Sistema Inteligente pretende-se uma adaptação de princípios do Método Clínico Piagetiano para a concepção e operacionalização do sistema, aplicando-se os desafios e, com isto, observando-se o processo de construção realizada pelo usuário, assim como o entendimento de suas próprias ações e decisões, por tomadas de consciência.

O IOCS deverá compor um "pool" de software de uma nova plataforma-ambiente que é a integração do aplicativo NETCLASS2 desenvolvido pelo Laboratório Sistemas Inteligentes LSI/UFMA (Labidi et al., 2000) e com o aplicativo CIVITAS3 do Laboratório de Estudos em Linguagem, Interação e Cognição - LELIC/UFRGS, (Axt et al., 2000).

\section{Sistema de Desafios Abertos Inteligentes - (IOCS)}

Esta proposta está em fase de implementação. Propõe um sistema inovador na aplicação da tecnologia multiagentes porque enfatiza a pró-atividade nos dois sentidos, isto é, a interação entre o sujeito (usuário) e o objeto (sistema) possibilitando situações problematizadoras por meio de estratégias baseadas em provas e adaptações da metodologia clínica piagetiana trabalhando, assim, o entendimento e a construção por patamares de conhecimento.

Para Piaget, o desenvolvimento do conhecimento é concebido por quatro fatores: o primeiro, a maturação; o segundo, o papel da experiência; o terceiro, a transmissão social e o quarto ele chamou de equilibração. Todos são de suma importância, mas o foco deste artigo encontra-se no fator experiência, ou seja, nos efeitos do ambiente físico na estrutura da inteligência e no fator equilibração (processos que se auto-regulam mediante uma compensação progressiva, isto é, um processo ativo).

A base funcional do IOCS é formada pelo tripé: Sistema, Agente Estrategista e Usuário.

\footnotetext{
${ }^{1}$ Para esta pesquisa os desafios possuem predicativos de objetos de aprendizagem.

${ }^{2}$ NETCLASS é um ambiente ensino-aprendizagem que utiliza técnicas de inteligência artificial.

${ }^{3}$ CIVITAS é um software e metodologia de criação de uma cidade virtual em cooperação. 
O Sistema - Arquitetura multiagentes desenvolvida usando a linguagem Java ${ }^{\circledR}$ (Java, 2004), tem como sua função principal a aplicação e interação dos desafios com o usuário. Tais Desafios são programados na linguagem Action Script (Action Script, 2004), com predicativos de objetos de aprendizagem4, os quais são baseados nas provas de Piaget (1995).

O Agente Estrategista - Agente responsável por aplicar os desafios, simulando o papel de experimentador5 virtual, possibilitando a interação do Usuário com o Sistema.

O Usuário - constituirá novos entendimentos da relação "usuário x jogo", adquirindo tomadas de consciência do jogo, por meio dos princípios da abstração reflexionante aplicada pelo Sistema por meio do Agente Estrategista, gerando, assim, possibilidades para chegar até um meta-entendimento.

O IOCS acaba por constituir um sistema multiagente que apóia os processos de aprendizagem. Sua utilização, espera-se, ser viável em ambientes virtuais de aprendizagem, como apoio para o desenvolvimento de estratégias pedagógicas.

\subsection{Sistema}

O Sistema tem como base a teoria de Jean Piaget, principalmente a Abstração Reflexionante. Os resultados esperados devem enfatizar a pró-atividade nos dois sentidos, aferindo ao usuário um progresso significativo na sua capacidade cognitiva, propiciando a compreensão e a construção de novos patamares de conhecimento.

Para isso é necessário desenvolver abstrações cada vez mais reflexionantes as quais se apóiam "sobre objetos procedentes de ações ou operações do sujeito e transferem a um plano superior (de organização) tudo o que foi tirado de um nível inferior de atividade" (Piaget, 1995), produzindo novas formas de conhecimento.

A abstração reflexionante comporta sempre dois aspectos:

A reflexão - ato mental de reconstrução e reorganização sobre o patamar superior daquilo que foi assim transferido do inferior.

O reflexionamento - transpõe-se a um plano superior o que colhe no patamar precedente;

A Figura 1 apresenta o processo de abstração reflexionante, formando os patamares.

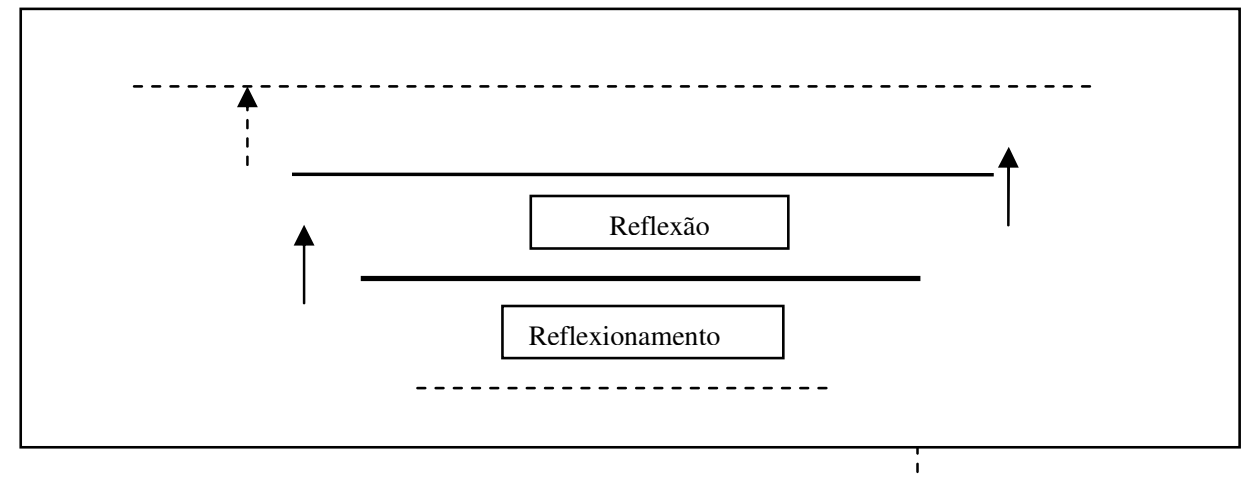

Figura 1 - Processo da Abstração Reflexionante

Tais formas abstraídas ou depreendidas a partir das atividades cognitivas do sujeito (esquemas ou coordenações de ações, operações estruturas etc.), pela retirada de certas características mais constantes, são, por sua vez, utilizadas para outras finalidades (novas adaptações, novos problemas).

\subsection{Desafios Abertos}

\footnotetext{
4 Objeto de Aprendizagem é qualquer recurso digital que possa ser reutilizado e ajude na aprendizagem.

${ }^{5}$ Experimentador é quem aplica o método clínico e desenvolve as seguintes ações: saber observar, ou seja, deixar a criança falar, não desviar nada, não esgotar nada e saber buscar algo de preciso, como uma tarefa com hipóteses pré-determinadas, propondo contra-argumentações, perguntas de antecipações, etc. 
Os desafios são objetos de aprendizagem. Serão construídos baseados nas provas de Piaget, dividas em três categorias:

- Abstração das relações lógico-aritméticas - Neste desafio será trabalhada o raciocínio sobre hipótese independente do conteúdo que tem como elementos básicos quatro transformações chamadas INRC - (I-identidade, N-negação, Rreciprocidade e C-correlativa).

- Abstrações da ordem das relações espaciais - Fazem parte das estruturas lógico-aritméticas e são formas elementares de seriações ou de sucessões de movimentos de maneira a precisar as perspectivas, parte das abstrações. Nos desafios, por exemplo, haverá uma seqüência lógica. Para que o usuário possa completá-la, terá que compreender a lógica aplicada, parte do processo de abstração reflexionante.

- Abstração das relações espaciais - Neste item será reproduzida a existência de um espaço dos objetos, como também uma geometria do sujeito utilizando diagonais, certos movimentos cíclicos e diversas formas de translações e de rotações. Para se reproduzir estas formas o mais próximo da realidade será proposto o uso de linguagem de programação que tenha capacidade de gerar movimentos em 3D, como: Java 3D (2005) ou Virtual Reality Model Language - VRML, (2005).

\subsection{Os estágios}

O Sistema possui três estágios fomentando as Abstrações Reflexionantes, dinamizando o processo de equilibração: estágio de observação estágio de fazer para compreender e estágio de compreender no pensamento.

Os desafios serão estruturados em níveis, isto é, não é possível alcançar o segundo nível a não ser que o equilíbrio tenha sido alcançado no primeiro nível. O equilíbrio do terceiro nível só se torna possível quando o equilíbrio do segundo tenha sido alcançado e assim por diante.

\subsection{Agente Estrategista}

Há uma característica importante do Sistema que está em consonância com os padrões do Método Clínico Piagetiano: a possibilidade de uma aplicação a objetos de aprendizagem a partir de uma metodologia de estudo cognitivo. No Sistema, pretende-se simular o papel do experimentador com o objetivo de construção de novos patamares, inclusive, por tomadas de consciência.

\subsection{Usuário}

"O bom experimentador deve efetivamente, reunir duas qualidades muitas vezes incompatíveis: saber observar, ou seja, deixar a criança falar, não desviar nada, não esgotar nada e, ao mesmo tempo, saber buscar algo de preciso, ter a cada instante uma hipótese de trabalho, uma teoria, verdadeira ou falsa, para controlar." (Piaget, 1993, p. 17)

Dentro da arquitetura do IOCS, o Usuário toma duas posições, ou seja, como agente humano do sistema, através de uma argumentação aberta do software Forchat, pode expressar suas idéias, como resolveu tal desafio e mediando a interação com outros usuários, através do envio de sugestões, integrando-se ao sistema do IOCS, e como Usuário, propriamente dito, sendo assim, o Sujeito da ação, que irá interagir com o Sistema, por meios dos desafios e sendo problematizado pelo Agente Estrategista.

\section{Arquitetura do IOCS}


Nesta seção, apresentaremos a arquitetura do IOCS. Na presente proposta, o experimentador será simulado pelos agentes inteligentes que buscam padrões no seu usuário, a fim de selecionar estratégias desafiadoras. A plataforma será desenvolvida utilizando a linguagem Java (2004).

Os agentes que compõem o Sistema são, conforme Figura 2, abaixo:

- Usuário - que interage com o Sistema e com outros Usuários.

- Agente de Protocolo - é o agente responsável por registrar as ações do Usuário, obtidos pela Interface, durante o processo de construção da solução do desafio, visando, com isso, o registro de informações e da evolução cognitiva do Usuário pelo Sistema. Estes registros poderão ser utilizados em situações futuras pelo próprio Sistema IOCS e outros sistemas afins, para análise dos dados.

- Agente de Modelagem - realiza a modelagem das informações, criando um perfil do Usuário para o Agente Estrategista, através do Agente Desafiador, possibilitando com que este obtenha dados para uma posterior tomada de decisão. As cinco reações da criança, observadas pelo exame clínico de Piaget (1926), serão adaptadas a fim de construir perfis do Usuário possíveis de processamento para o Sistema IOCS:

- Não-importismo - quando o Usuário não demonstra esforço algum de resposta;

- Fabulação - quando o Usuário responde sem refletir, inventando uma situação não contextualizada no desafio;

- Crença Sugerida - quando o Usuário responde ao desafio sugestionado pelo Agente Estrategista sem refletir os detalhes contidos na problematização, testemunhando, assim, apenas a sua incompreensão em relação ao mesmo;

- Crença Desencadeada - quando o Usuário responde ao desafio por meio de seus próprios conhecimentos anteriormente adquiridos - a resposta é um produto original de seu pensamento -, porém, necessariamente influenciado pelas questões que o Sistema irá fazer, forçando-o a raciocinar e a sistematizar o seu conhecimento em certa direção. Ou seja, o raciocínio do Usuário foi desencadeado "artificialmente" (não sugerido nem, contudo espontâneo) devido a uma situação controlada experimentalmente pelo Agente Estrategista enfim, produto de raciocínio feito sob comando, mas com recursos de materiais (conhecimentos próprios, imagens mentais esquemas motores, preligações sincréticas entre outros) e instrumentos lógicos (estrutura do raciocínio, orientações do pensamento, hábitos intelectuais entre outros) originários dele mesmo;

- Crença Espontânea - quando o Usuário responde imediatamente ao desafio, não necessitando raciocinar, já que o desafio formulado não lhe é novo, sendo a resposta fruto de uma reflexão anterior e original (abstração reflexionante), ou seja, desenvolvida pelo próprio Usuário em suas situações naturais de realidade de vida. 


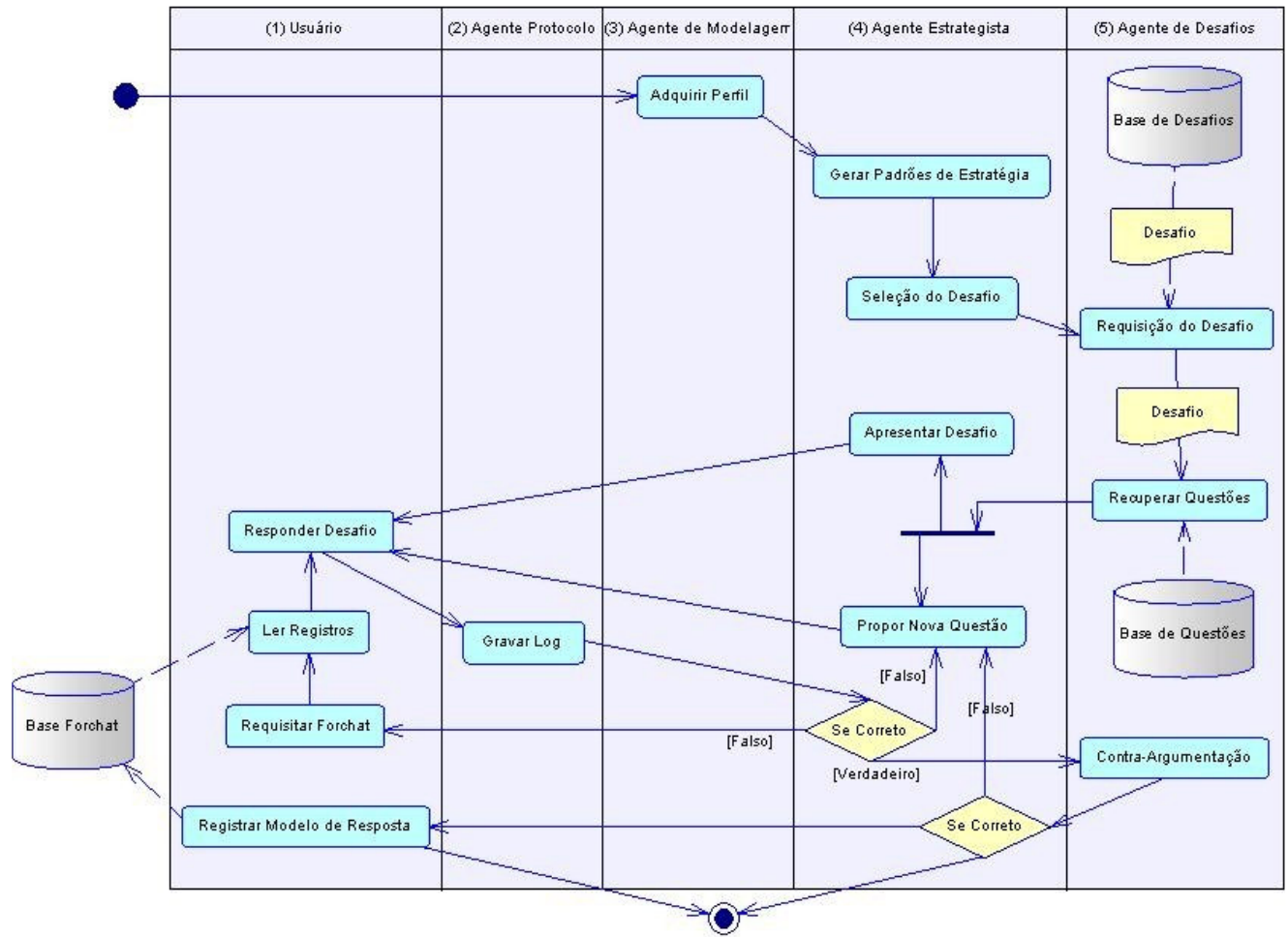

Figura 2 - Arquitetura IOCS

- Agente Estrategista - tem ação estratégica dentro do Sistema, já que reúne funcionalidades de controle para a interação. A partir do perfil do Usuário, informado pelo Agente de Modelagem, gera um padrão de estratégia contido em umas das etapas da Interface (ver tópico 4.3), informando-o ao Agente de Desafios; posteriormente, apresenta o desafio ao Usuário. Também poderá propor nova questão (contra-argumentação) ao Usuário ,de acordo com o desafio já selecionado pelo Agente de Desafios, criando um processo de problematização a fim de provocar desequilíbrios cognitivos no Usuário, simulando, assim, o papel do experimentador, tornando-se, de acordo com a proposta deste estudo, um Experimentador Virtual.

- Agente de Desafios - gerencia as bases de dados dos desafios e questões (objetos de aprendizagem). De acordo com a etapa fornecida pelo Agente Estrategista, requisita o desafio que deverá ser apresentado ao Usuário. Base de Desafios - Local de armazenamento dos desafios e seus estágios, os quais serão acionados em consonância com as requisições do Agente Estrategista. Base de Questões - Local de armazenamento de perguntas ou questões. Cada grupo de questões tem um nível de associação com as etapas dos desafios; com isso, não haverá a repetição de questões nem questões inadequadas ao desafio a ser aplicado. Exemplos: O que escrevi lá? Como são ordenadas?

\section{Caso de uso}

Algumas visões em termos de modelagem foram criadas, para o Sistema de Aprendizagem proposto, baseadas nos estudos de Piaget. Os casos de uso e diagramas foram criados por meio da linguagem UML (Linguagem de Modelagem Unificada). O sistema foi dividido em três casos de uso, são eles: "Criação da Modelagem do Usuário", "Escolha de Estratégia", "Realizar Desafios". Cada um desses complementa as ações tomadas pelo Sistema para a realização das atividades que permitem analisar o usuário. Para alimentar as decisões tomadas por esses agentes, foram criadas duas bases de dados: a "Base de Desafios" e a "Base de Estratégia”. A visão geral dos casos de uso é mostrada na Figura 3. 


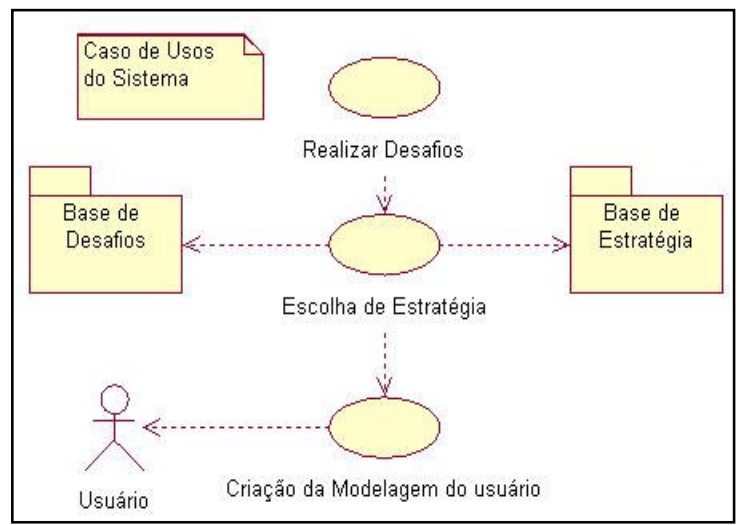

Figura 3 - Casos de Uso do Sistema

O caso de uso "Criação da Modelagem do Usuário" possui a responsabilidade de interação com o usuário para a obtenção de informações que sirvam de indicativo para a criação de um perfil do usuário. A criação da modelagem desse usuário poderá ser realizada utilizando técnicas de redes neurais ou simplesmente interagindo (sistema) com o usuário por meio de perguntas. As informações do perfil do Usuário serão posteriormente enviadas ao Agente Estrategista.

Fundamentada nesses itens, a Figura 4 mostra o agente de modelagem realizando a coleta das informações do usuário. De acordo com a análise do diagrama de classe da Figura 3, o agente de modelagem controla as classes envolvidas em obter o perfil do usuário em uma relação de dependência. Dependendo do usuário, as informações podem vir de todas, de algumas, ou de somente uma classe de armazenamento (Não-importismo, Fabulação, Crença Sugerida, Crença Desencadeada, Crença Espontânea).

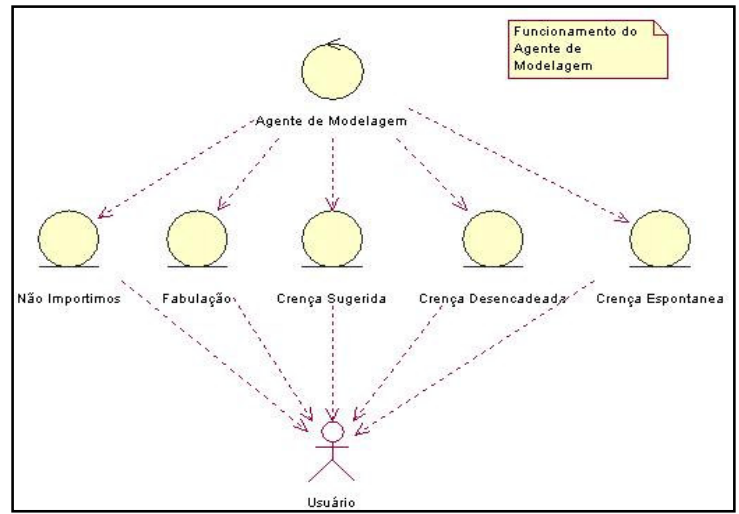

Figura 4 - Funcionamento Criação do Agente de Modelagem

Todas as classes de armazenamento que irão obter as informações do usuário dependem diretamente dele. As setas em direção ao usuário representam essa situação de dependência.

Observa-se no diagrama de colaboração da "Modelagem do Usuário" (ver Figura 5) que o agente de controle de modelagem do agente inicialmente carrega as informações do banco de desafios; escolhido o desafio, o controle do agente envia o desafio ao usuário por meio de uma interface do sistema.

O Usuário realiza o desafio e, após essa etapa, o agente por meio do seu centro de controle analisa as informações coletadas advindas das ações do usuário para em seguida armazená-las em uma base de informações que servirá de histórico das atividades do usuário. Essas informações serão arquivadas por meio de um agente do sistema denominado de "Agente de Protocolo". Após a realização do arquivamento dessa informação, a informação será transmitida ao agente estrategista. 


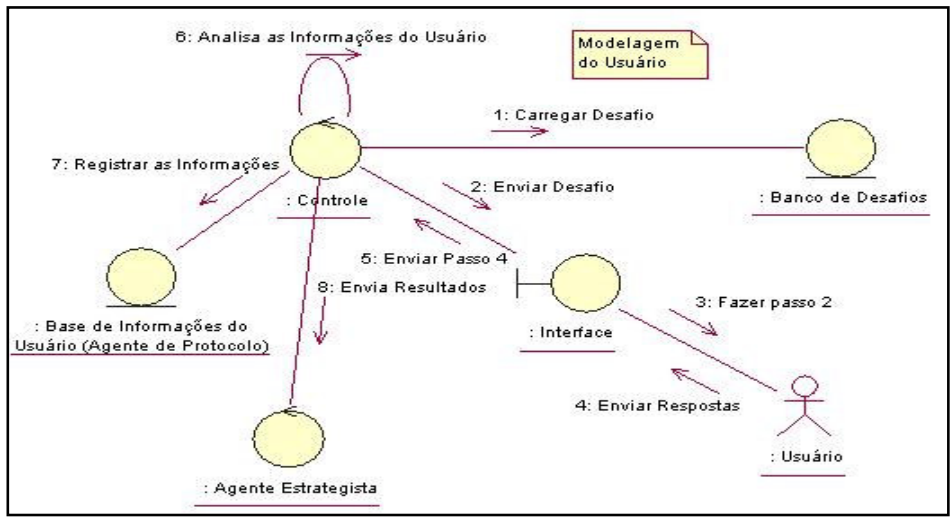

Figura 5 - Diagrama de Colaboração do Caso de Uso "Modelagem do Usuário"

O Agente estrategista (Figura 6) de posse das informações provenientes do Agente de modelagem realiza as operações básicas de verificação de usuário bem como recuperação das informações anteriores cujo local de deposito é a "Base de Informações do Usuário". De posse dessas informações, o controle do agente estrategista seleciona a estratégia adequada a qual se encontrará armazenada na base de questões. Carregada a estratégia, o passo seguinte será carregar o desafio, função do agente de desafio.

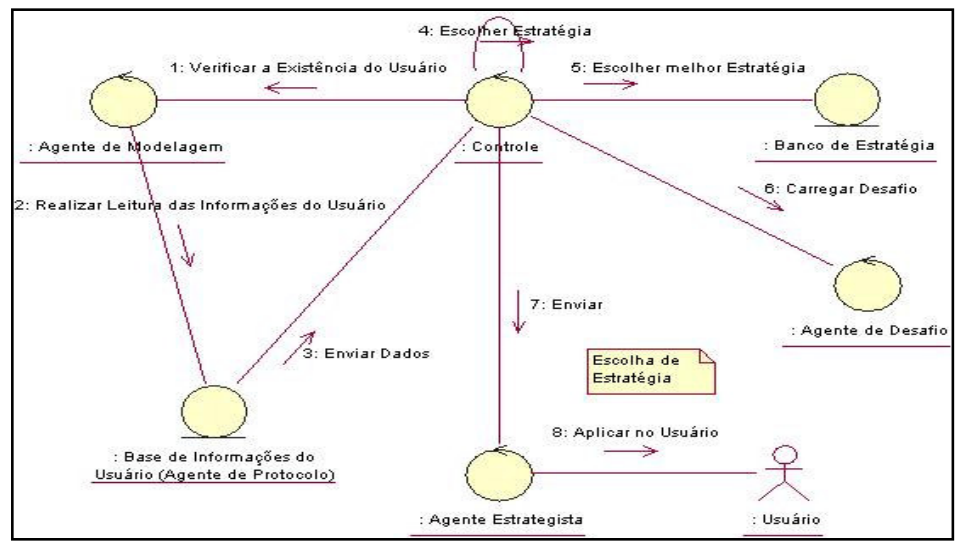

Figura 6 - Diagrama de Colaboração do Caso de Uso "Escolha de Estratégia"

Realizadas as operações de seleção de estratégia e de desafio como é mostrado acima no diagrama "Escolha de Estratégia", o controle do agente envia os desafios e as intervenções para a interação com o usuário. É importante salientar que todos os diagramas (classe, colaboração, outros) utilizados na elaboração do sistema possuem cenários alternativos nos quais o sistema possui outros modos de alcançar os seus objetivos ou mesmo reiniciar os passos anteriores com outros cenários em que possam alcançar seus objetivos iniciais. Uma analogia a esse processo seria a utilização do procedimento de decisão "SE" da programação de computadores. Isto é, caso uma etapa não funcione a contento, outras ações tentarão conseguir retornar ao objetivo.

Em outras palavras, o objetivo do caso de uso, por meio do agente estrategista, é selecionar a ação estratégica adequada possibilitando uma situação em que se desequilibre cognitivamente o usuário. Nesse momento, o sistema passa a assumir o papel de experimentador.

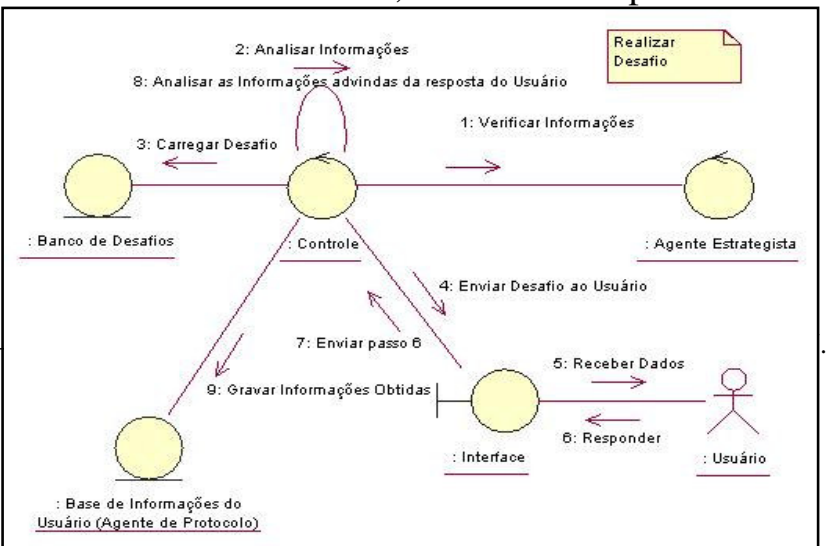


Figura 7 - Diagrama de Colaboração do Caso de Uso "Realizar Desafios"

O último caso de uso foi nomeado de "Realizar Desafios" cujo objetivo é fazer com que o agente desafiador possa selecionar ou buscar um desafio na base de desafios. De acordo com o diagrama de colaboração (Figura 7), o agente de desafios recebe as informações do Agente Estrategista para então realizar uma análise. Analisadas as informações, o desafio é carregado do "Banco de Desafios". Carregado o desafio, a etapa seguinte é enviá-lo para a interface do sistema. Após o desafio ser realizado pelo Usuário, vários procedimentos são disparados à análise das ações do usuário, não tendo como parâmetro o acerto ou o erro.

As informações recebidas na interface a partir das ações do usuário alimentam a "Base de Informações do usuário" cuja responsabilidade de gerenciar essas informações é do agente de protocolo.

As bases de informações não são estáticas, pois são constantemente atualizados de acordo com as ações do usuário a cada novos desafios. Cada estratégia e desafio são escolhidos de acordo com as características individuais dos usuários do sistema.

Diferentemente de outros sistemas, cuja preocupação é a de verificar somente se o usuário acertou ou não a questão solicitada, o sistema de aprendizagem proposto coloca em prática pressupostos pedagógicos construtivistas com uma preocupação do porquê e do como se realiza o processo de aprendizagem.

\section{Conclusão}

A proposta desta pesquisa é buscar, por meio de um sistema inteligente com incremento lúdico desenvolver atividades ligadas à aprendizagem, promovendo o desenvolvimento do conhecimento como um processo espontâneo, ou seja, um processo com auto-regulação. Para isso, o sujeito tem que ser ativo, interagindo com o objeto pois conseqüentemente defrontar-se-á com perturbações externas e internas e reagirá com o fim de compensar e tenderá para o equilíbrio como processo ativo. Este processo de equilibração toma forma de uma sucessão de níveis de equilíbrios, situação fundamental trabalhada pelo sistema e pelos objetos de aprendizagem (desafios). Outra situação é que o sistema irá mapear a ação do usuário, possibilitando o registro da evolução do ganho cognitivo do mesmo. Também possibilitará situações em que usuário mostre, por uma resposta pessoal e aberta, por meio de um software, $o$ Forchat, como chegou ao seu entendimento, não se preocupando se está certo ou errado.

Finalmente, o que se pretende é desenvolver um sistema inteligente adaptativo e construtivista, propiciando ao sujeito a ação sobre o objeto e não somente um sistema reativo, comportamentista, de estímulo-resposta. Esse sistema poderia ainda se beneficiar de um aporte teórico construído nas relações dialógicas, através da interação entre sujeitos, entre objetos, entre sujeitos e objetos, cf. Bakhtin (2000), para explorar efeito de potência das tecnologias digitais enquanto dispositivo para novos agenciamentos coletivos de enunciação (no lúdico), que privilegiassem a multiplicidade na produção de sentidos e significações em posições de autoria. (Axt et al., 2004).

\section{Referências}

ACTINSCRIPTS, 2004 - http://www.actionscripts.org/

AXT, M. (2000) "Tecnologia na Educação, Tecnologia para Educação: um texto em construção". Informática na educação: teoria \& prática, v. 3, n. 1, p. 51-62, set.

AXT, M. et al. (2004). "Sentido e Autoria: o lugar da multiplicidade na formação docente". Laboratório de Estudos em Linguagem, Interação e Cognição - LELIC/UFRGS. 
BAKHTIN, M. (2000) "Estética da Criação Verbal". São Paulo: Martins Fontes.

BASTOS FILHO, O.. C., Axt, M., Fonseca, L. C., Labidi, S., Guimarães, L. Nunes, Thomaz, A. R., Nascimento E. (2004) "Jogos inteligentes como sistemas abertos: singularizando as possibilidades de interação com lógicos". Revista Novas Tecnologias na Educação, ISSN 1679-1916. http://www.cinted.ufrgs.br/renote/nov2004/artigos/othon_jogos_inteligentes.pdf.

JAVA, (2005) http://java.sun.com/

LABIDI, S; Silva, J. C.; Coutinho, L.R.; Costa e. B. (2000) "Agent-based tutoring system for supporting cooperative and distant learning". In Proceedings of the International Conference on Computers and Advanced Technology in Education (CATE'2000).

MONTANGERO, J. and Maurice-Naville, D. (1994) "Piaget ou l'intelligence en marche." Liège, Mardag.

PIAGET, J. (1926) “A representação do mundo na criança”. Rio de Janeiro: Record.

PIAGET, J. (1976a) "A Equilibração das Estruturas Cognitivas. Problema central do desenvolvimento.” Trad. Álvaro Cabral. Rio de Janeiro: Zahar.

PIAGET, J. (1971) “A Formação do Símbolo na Criança. Imitação, jogo e sonho, imagem e representação”.Trad. Alvaro Cabral. Rio de Janeiro: Zahar.

PIAGET, J. (1995) “Abstração Reflexionante: Relações lógico-aritméticas e ordem das relações espaciais". Trad. Fernando Becker e Petronilha G. da Silva, Porto Alegre: Artes Médicas.

PIAGET, J. (1976b.) "Da Lógica da Criança à Lógica do Adolescente". São Paulo: Pioneira.

PIAGET, J. Fazer e Compreender. Trad. Cristina L. de P. Leite. São Paulo: Melhoramentos; EDUSP, 1978. 186p.

PIAGET J. et al. (1977) "A Tomada da Consciência”. Trad. Edson B. de Souza. São Paulo: Melhoramentos e EDUSP,. 211p.

RIPPLE, R e Rockcastle, V. (1964) "Piaget rediscovered". Cornell University.

VRML, (2004). Full details of the history and specifications can be seen at the VRML repository at http://www.sdsc.edu/vrml/. 